\title{
Biologia e parasitologia do morcego insetívoro aéreo Myotis nigricans (Schinz, 1821) (Chiroptera, Vespertilionidae) no município do Rio de Janeiro, Rio de Janeiro, Brasil Biology and parasitology of the aerial insectivorous bat Myotis nigricans (Schinz, 1821) (Chiroptera, Vespertilionidae) in the city of Rio de Janeiro, Rio de Janeiro, Brazil
}

\author{
Raissa da Silva Dias Oliveira' | Shirley Seixas Pereira da Silval (1) | \\ Patrícia Gonçalves Guedes (D) | Juliana Cardoso de Almeidal, II, III (D) \\ IInstituto Resgatando o Verde. Rio de Janeiro, Rio de Janeiro, Brasil \\ "Universidade Iguaçu. Departamento de Ciências Biológicas e da Saúde. Nova Iguaçu, Rio de Janeiro, Brasil \\ IIICentro Universitário Geraldo di Biase. Volta Redonda, Rio de Janeiro, Brasil
}

\begin{abstract}
Resumo: Myotis nigricans (Schinz, 1821) é um morcego insetívoro aéreo amplamente distribuído na região neotropical, encontrado em todos os biomas brasileiros e adaptado aos centros urbanos. Apresentamos dados bionômicos, reprodutivos, de dieta e parasitológicos de 28 exemplares de Myotis nigricans capturados em áreas de Mata Atlântica no município do Rio de Janeiro, Sudeste do Brasil, com o propósito de oferecer mais informações sobre as interações ecológicas, especialmente aquelas oriundas de parques urbanos. Os exemplares apresentaram medidas semelhantes às descritas para a espécie, estando sexualmente ativos na primavera (outubro). Fragmentos de insetos das ordens Diptera, Coleoptera, Hemiptera e Lepidoptera foram encontrados nas amostras fecais examinadas. Cinco indivíduos estavam infestados por Basilia sp. (Diptera, Nycteribiidae), e helmintos das classes Trematoda e Cestoda foram encontrados no trato gastrointestinal, estes constituindo o primeiro registro para M. nigricans. Apesar da ampla distribuição desta espécie, os dados sobre bionomia e interações ecológicas são fragmentados e pontuais. Desta forma, os resultados aqui apresentados reforçam a importância da realização de acompanhamentos sistemáticos para a compreensão das relações estabelecidas por esses animais em ambientes florestados e antropizados.
\end{abstract}

Palavras-chave: Atividade reprodutiva. Bionomia. Insetivoria. Mata Atlântica. Sinantropia.

Abstract: Myotis nigricans (Schinz, 1821) is an aerial insectivorous bat widely distributed in the neotropical region, found in all Brazilian biomes and adapted to urban centers. We present bionomic, reproductive, dietetic and parasitological data from 28 specimens of Myotis nigricans captured in Atlantic Forest areas in the city of Rio de Janeiro, southeastern Brazil, in order to provide more information on ecological interactions of these animals, especially those from urban parks. The specimens presented measurements similar to those described for the species, being sexually active in spring (October). Insect fragments of the orders Diptera, Coleoptera, Hemiptera and Lepidoptera were found in the stool samples examined. Five individuals were infested with Basilia sp. (Diptera, Nycteribiidae), and helminths of the Trematoda and Cestoda classes were found in the gastrointestinal tract, the latter constituting the first record for $M$. nigricans. Despite the widespread distribution of this species, data on bionomy and ecological interactions are fragmented and punctual. Thus, the results presented here reinforce the importance of carrying out systematic follow-ups to understand the relationships established by these animals in forested and anthropized environments.

Keywords: Reproductive activity. Bionomy. Insectivory. Atlantic Forest. Synanthropy.

OLIVEIRA, R. S. D., S. S. P. SILVA, P. G. GUEDES \& J. C. ALMEIDA, 2020. Biologia e parasitologia do morcego insetívoro aéreo Myotis nigricans (Schinz, 1821) (Chiroptera, Vespertilionidae) no município do Rio de Janeiro, Rio de Janeiro, Brasil. Boletim do Museu Paraense Emílio Goeldi. Ciências Naturais 15(3): 633-642. DOI: http://doi.org/10.46357/bcnaturais.v15i3.247.

Autora para correspondência: Shirley Seixas Pereira da Silva. Rua Tirol, 536 sala 609, Freguesia, Jacarepaguá. Rio de Janeiro, RJ, Brasil. CEP 22750-009 (batshirley@gmail.com).

Recebido em 20/12/2019

Aprovado em 04/11/2020

Responsabilidade editorial: Alexandra Maria Ramos Bezerra
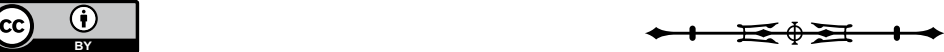


\section{INTRODUÇÃO}

A Mata Atlântica do estado do Rio de Janeiro, Sudeste do Brasil, abriga grande diversidade biológica, e as modificações propiciadas pela crescente urbanização resultam em redução significativa desta biodiversidade (Esbérard, 2003; Rocha et al., 2003). Segundo Lima (2008), no ambiente urbano, os fragmentos florestais são verdadeiras "ilhas verdes" - como parques municipais, bosques e praças - cercadas por habitações humanas. $\bigcirc$ levantamento da quiropterofauna nessas áreas remanescentes é fundamental para analisar a diversidade em meio urbano e compreender as interações estabelecidas com outras espécies, incluindo o homem.

Morcegos vespertilionídeos mostram-se mais tolerantes às modificações ambientais causadas pelo homem, ocupando construções humanas como forma de abrigo e utilizando a iluminação pública como fonte de atração de insetos (Rydell, 1992; Bredt \& Uieda, 1996). O gênero Myotis Knaup, 1829, com distribuição cosmopolita, está representado no Brasil por oito espécies (Nogueira et al., 2018): Myotis albescens (E. Geoffroy, 1806), Myotis izecksohni Moratelli, Peracchi, Dias \& Oliveira, 2011, Myotis lavali Moratelli, Peracchi, Dias \& Oliveira, 2011, Myotis levis (I. Geoffroy, 1824), Myotis nigricans (Schinz, 1821), Myotis riparius Handley, 1960, Myotis ruber (E. Geoffroy, 1806) e Myotis simus Thomas, 1901.

Myotis nigricans pertence à guilda dos insetívoros aéreos e tem distribuição em todas as regiões brasileiras, não sendo registrado apenas nos estados do Acre, Alagoas, Piauí e Rondônia (Aguirre et al., 2003; Reis et al., 2013). No estado do Rio de Janeiro, essa espécie foi registrada nos principais remanescentes florestais de Mata Atlântica (Dias et al., 2002, 2010; Esbérard, 2003; Dias \& Peracchi, 2008; Modesto et al., 2008a, 2008b; Peracchi \& Nogueira, 2010), construções humanas e em unidades de conservação (UC) no município do Rio de Janeiro (Pinto, 2008; Silva et al., 2010; Esbérard et al., 2014). Apesar dos morcegos desta espécie terem ampla distribuição e serem conhecidos por sua dieta insetívora, há poucas informações sobre aspectos da biologia desses animais, como a composição da dieta e da reprodução, além da interação com parasitas. O presente estudo tem como objetivo apresentar informações sobre indivíduos de Myotis nigricans em ambientes florestais e em área urbana, com vistas a ampliar o conhecimento sobre espécies sinantrópicas que habitam o interior e o entorno de remanescentes florestais no município do Rio de Janeiro, Rio de Janeiro, Brasil.

\section{MATERIAL E MÉTODOS}

\section{ÁREA DE ESTUDO}

As atividades de campo para coleta de morcegos foram realizadas em um período de 25 anos - de março de 1994 a outubro de 2004 no Parque Estadual da

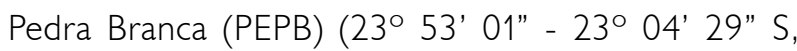

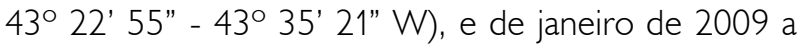
outubro de 2019 nas seguintes unidades de conservação: Parque Natural Municipal Chico Mendes (PNMCM)

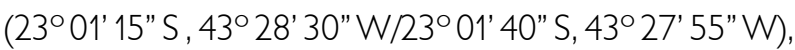
Parque Natural Municipal da Freguesia(PNMF)(22 $56^{\prime} 49^{\prime \prime} \mathrm{S}$, $43^{\circ} 20^{\prime} 35^{\prime \prime}$ W) e Parque Natural Municipal Bosque da

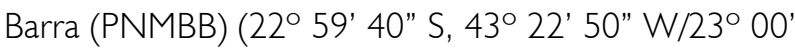

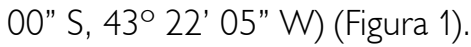

O Parque Natural Municipal Chico Mendes possui 43,64 ha e a vegetação é constituída por formações pioneiras, predominando a mata de restinga (IBGE, 2012). O Parque Natural Municipal Freguesia, conhecido como 'Bosque da Freguesia', possui 30,33 ha e vegetação em estágio inicial e médio de regeneração, pertencente às formações pioneiras e à floresta ombrófila densa de baixada (IBGE, 2012). O Parque Natural Municipal Bosque da Barra, conhecido como 'Bosque da Barra', está localizado em uma região conhecida como 'Baixada de Jacarepaguá', possui 54,398 hectares e é composto por vegetações típicas das matas de restinga (IBGE, 2012). O Parque Estadual da Pedra Branca, com 12.393,84 hectares, está situado no interior do

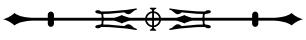




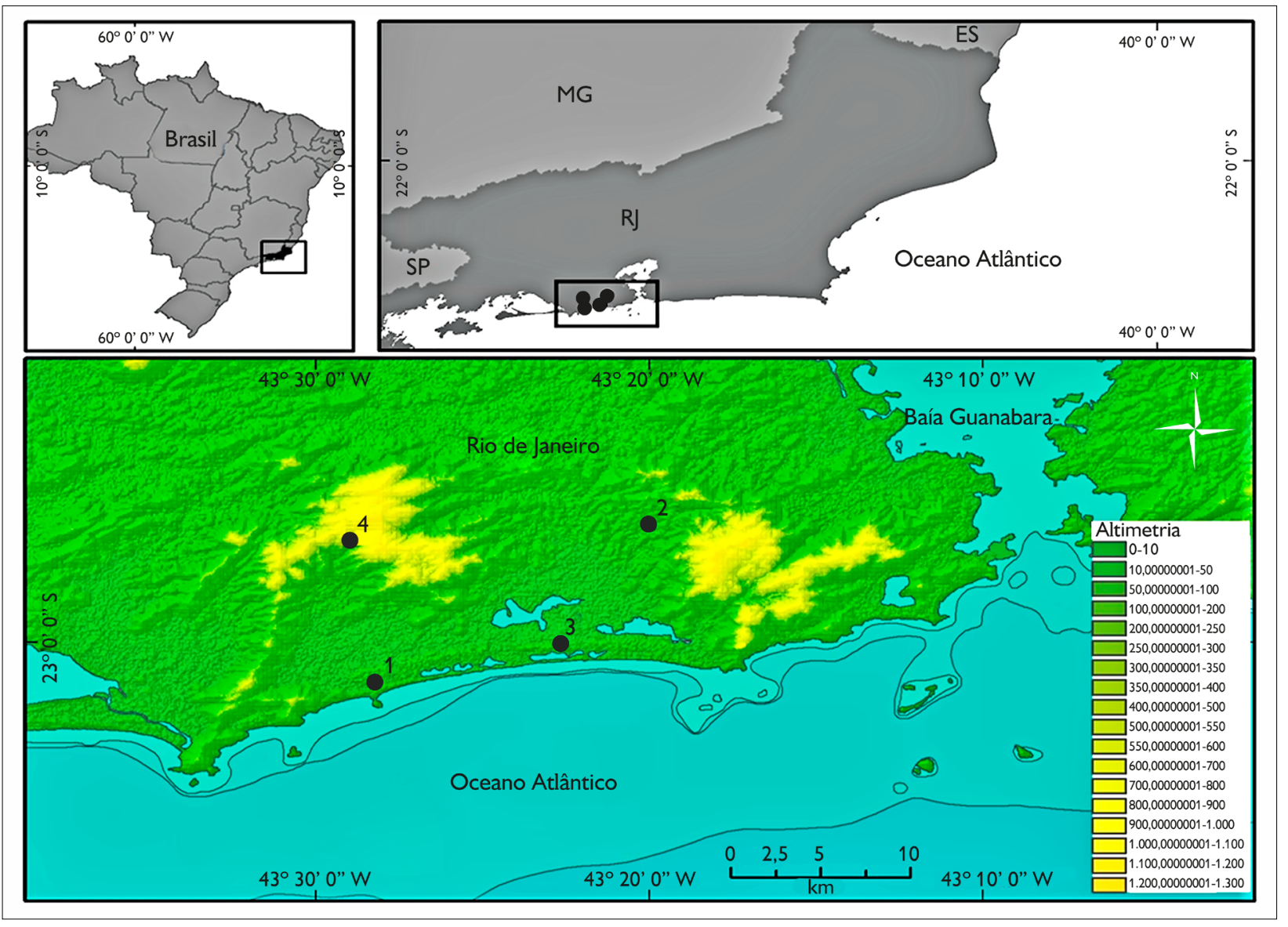

Figura 1. Localização das unidades de conservação no município do Rio de Janeiro, Rio de Janeiro, Brasil: 1 - Parque Natural Municipal Chico Mendes; 2 - Parque Natural Municipal da Freguesia; 3 - Parque Natural Municipal Bosque da Barra; 4 - Parque Estadual da Pedra Branca. Mapa: elaborado por Sergio Moreira (2018) e adaptado por Shirley S. P. Silva (2019).

Maciço da Pedra Branca, acima da cota de 100 metros na região metropolitana do município (INEA, 2015), apresentando vegetação constituída por Floresta Pluvial Baixo Montana, com trechos bem conservados e grande diversidade de formações, como Mata Pluvial Atlântica, Mata Atlântica Seca, Mata Atlântica Úmida, Mata de Encosta, Mata Seca Alta e Capoeiras (Rizzini, 1979).

\section{AMOSTRAGEM DOS ESPÉCIMES}

Os trabalhos de campo ocorreram em períodos distintos e houve diferença no esforço de captura para cada área analisada: $562 \mathrm{~m}^{2} / \mathrm{h}$ no Parque Natural Municipal Bosque da Barra, $5.400 \mathrm{~m}^{2} / \mathrm{h}$ no Parque Natural Municipal Chico
Mendes, $91.980 \mathrm{~m}^{2} / \mathrm{h}$ no Parque Natural Municipal da Freguesia e $36.225 \mathrm{~m}^{2} / \mathrm{h}$ no Parque Estadual da Pedra Branca, perfazendo um esforço de captura total de $134.167 \mathrm{~m}^{2} / \mathrm{h}$, calculado conforme Straube \& Bianconi (2002).

Os morcegos foram capturados com a utilização de cinco a oito redes de neblina de seis a nove metros de comprimento, armadas no sub-bosque, em trilhas existentes na mata ou sobre córregos, abertas antes do pôr do sol e fechadas após seis horas. Os indivíduos foram acondicionados em sacos de pano para obtenção de amostras fecais, guardadas em envelopes de papel individualizados. Dados biométricos padrão para morcegos (comprimentos total, do antebraço, do pé, da orelha, da

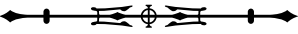


tíbia, do calcâneo e da cauda; veja Vizotto \& Taddei, 1973) foram obtidos com auxílio de paquímetro digital ( $\mathrm{mm})$, e a massa corporal (g) foi aferida com uso de dinamômetro. Os ectoparasitos encontrados na pelagem dos animais foram retirados com auxílio de pinças e acondicionados em microtubos plásticos com etanol $70 \%$, etiquetados.

A identificação dos fragmentos de insetos e ectoparasitos foi realizada com base em chaves taxonômicas e descrições disponíveis na literatura, como Carrera (1980), Whitaker (1988), Guerrero (1993) e Shiel et al. (1997). Os espécimes de morcegos preparados como material-testemunho tiveram o trato gastrointestinal removido e examinado sob estereomicroscópio (40x) para verificação da presença de endoparasitas, reportados no trabalho de Ferreira \& Brasil-Sato (1998).

\section{ANÁLISE DE HEMOPARASITOS}

Para avaliação da ocorrência de hemoparasitos no sangue periférico, amostras de sangue foram obtidas por punção da veia propatagial dos animais, com auxílio de agulhas hipodérmicas estéreis, após antissepsia com etanol 70\% (Baptista \& Esbérard, 1997). Após a punção venosa, foram preparados três a quatro esfregaços 'a fresco' por indivíduo. As lâminas hematológicas foram identificadas com auxílio de etiquetas adesivas, contendo o número de campo do indivíduo capturado, e foram embaladas individualmente com papel toalha, para posterior análise. Cinco indivíduos de $M$. nigricans do PEPB foram depositados como material-testemunho na Coleção Adriano Lúcio Peracchi (ALP), Universidade Federal Rural do Rio de Janeiro (UFRRJ), Seropédica, Rio de Janeiro: ALP 5727 (q), ALP 5742 (ठ)), ALP 5744 (す) ALP 5821 (ठ゚) e ALP 5861 (†). Espécimes em período reprodutivo e aqueles das demais localidades (PNMCM, PNMF e PNMBB) foram soltos ao final dos trabalhos de campo.

Em ambiente laboratorial, as lâminas hematológicas foram coradas pelo método ${ }^{\circledR}$ Panótico Rápido, sendo examinadas com auxilio de microscópio óptico (aumento de 1.000x), para verificar presença de hemoparasitos. As amostras fecais foram analisadas sob estereomicroscópio (40x), após ser realizada a separação, quantificação e identificação dos fragmentos de insetos, que foram montados em lâminas permanentes, posteriormente fotografados.

As amostras dos ectoparasitos estão depositadas na Coleção Acarológica do Instituto Oswaldo Cruz (IOC), da Fundação Oswaldo Cruz (FIOCRUZ), e os endoparasitos na Coleção Helmintológica do Instituto Oswaldo Cruz. Todos os trabalhos de campo foram realizados com autorização para atividades com finalidades científicas: números 11598-1, 11598-2, 11666-1, 18852-1, 22393-1, 22393-2, emitidos pelo Instituto Chico Mendes de Conservação da Biodiversidade (ICMBio), e 01/2009 (Proc. 14/000.047/2009), 12/2009 (Proc. 14/001.059/2009), 0613B, 0713B, 13_14B e 18_16B (Proc. 14/000.627/2013), emitidos pela prefeitura da cidade do Rio de Janeiro. O mapa de localização das áreas de estudo foi elaborado por meio do programa ArcView 3.2.

\section{RESULTADOS}

Ao longo de todo período de estudo, um total de 28 exemplares (10 fêmeas e 18 machos) de Myotis nigricans foram capturados (Figura 3A). A massa corporal (média $=4,4 \mathrm{~g}$; desvio padrão $(\mathrm{DP})=2,1$ ) variou de 2,0 a $11,0 \mathrm{~g}$ nos machos (média = 4,1 g) e 3,7 a 8,0 g nas fêmeas (média $=5,4 \mathrm{~g}$ ). A medida do antebraço (média $=34,1 \mathrm{~mm}$; $\mathrm{DP}=1,6)$ foi de 31,5 a $37,0 \mathrm{~mm}$ nos machos e 31,6 a $36,9 \mathrm{~mm}$ nas fêmeas. Na Figura 2 estão ilustradas as medidas corporais obtidas nos indivíduos. A Tabela 1 apresenta os intervalos dos valores obtidos em cada um dos parques.

Em relação à reprodução, apenas os morcegos capturados no PEPB estavam em período reprodutivo, pois foram observadas quatro fêmeas grávidas no mês de outubro. Os demais indivíduos foram considerados como sexualmente inativos, por não apresentarem evidências externas de estágio reprodutivo ou lactação. 


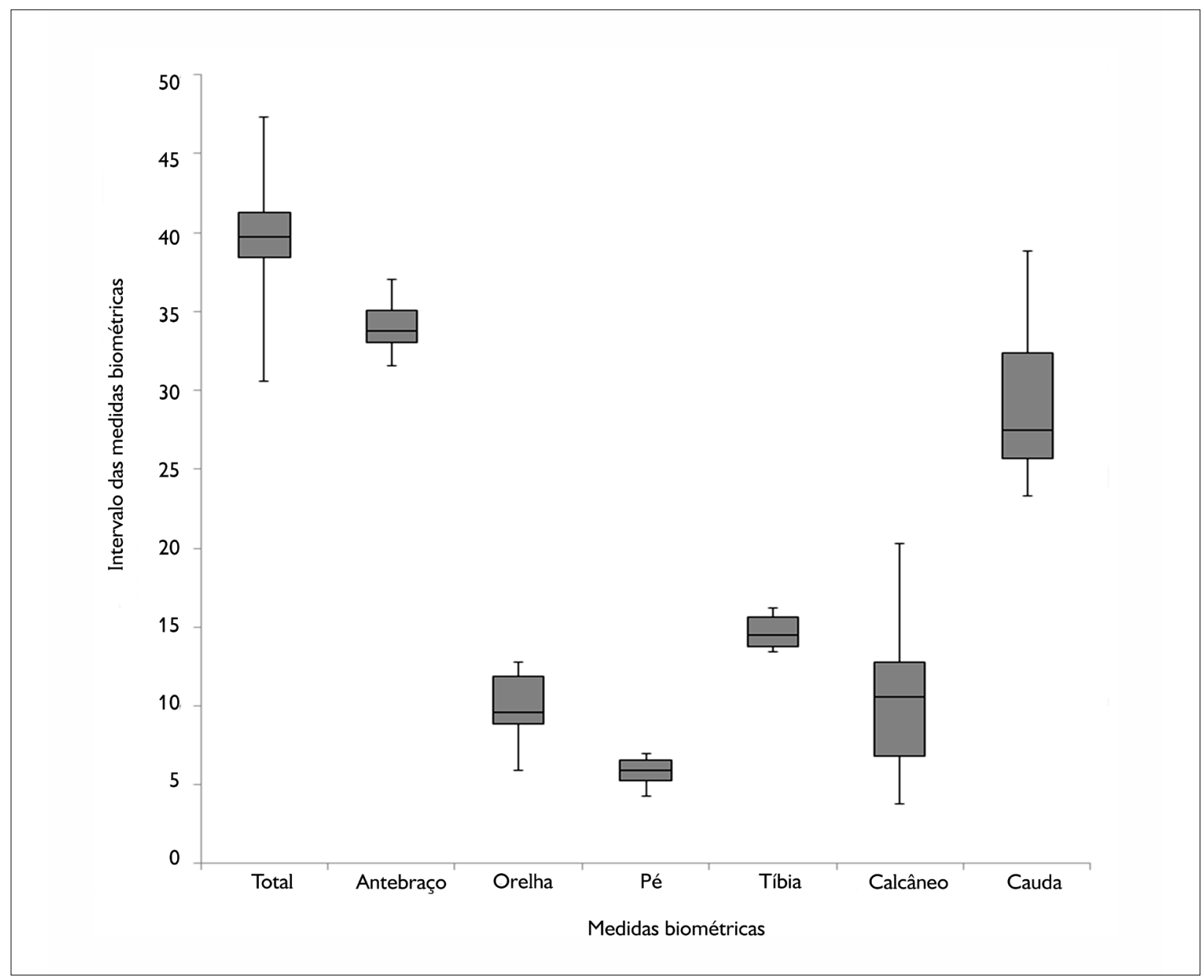

Figura 2. Intervalo das medidas biométricas obtidas em indivíduos de Myotis nigricans capturados em quatro unidades de conservação do município do Rio de Janeiro, Rio de Janeiro, Brasil (ver a Figura 1 paras as UC e a seção de Material e Métodos para as medidas biométricas tomadas).

O estudo da dieta foi realizado através da análise de oito amostras fecais obtidas no PNMF $(n=4)$ e no $\operatorname{PEPB}(n=4)$, que apresentaram somente fragmentos de insetos (Hexapoda), identificados como pertencentes às ordens Hemiptera, Diptera, Coleoptera e Lepidoptera (Figuras 3B-3H). Não foram encontrados resíduos alimentares no trato gastrointestinal dos indivíduos provenientes do PEPB.

Cinco indivíduos de $M$. nigricans capturados no PNMF apresentavam em sua pelagem ectoparasitos identificados como Basilia sp. (Diptera: Nycteribiidae) (Figura 3I). No trato gastrointestinal de dois indivíduos, foram encontrados helmintos trematódeos, reconhecidos como pertencentes à espécie Ochoterenatrema diminutum (Chandler, 1938) (Ferreira \& Brasil-Sato, 1998), e um Cestoda, que não pôde ser identificado devido à fragmentação do exemplar. Do total de exemplares de $M$. nigricans capturados no PNMF ( $n=12)$, foram analisadas lâminas hematológicas de cinco indivíduos, não sendo identificada a presença de hemoparasitos. 
Tabela 1. Massa corporal (em g) e comprimento de antebraço (em mm) dos indivíduos de Myotis nigricans capturados em unidades de conservação no município do Rio de Janeiro, Rio de Janeiro, Brasil, no período de 1994 a 2019. Legendas: PEPB = Parque Estadual Pedra Branca; PNMBB = Parque Natural Municipal Bosque da Barra; PNMF = Parque Natural Municipal da Freguesia; PNMCM = Parque Natural Municipal Chico Mendes; N = número de indivíduos analisados.

\begin{tabular}{|c|c|c|c|}
\hline Myotis nigricans & $N$ & Massa corporal (g) & Comprimento do antebraço $(\mathrm{mm})$ \\
\hline \multicolumn{4}{|l|}{ PEPB } \\
\hline Macho & 4 & $3,0-4,0$ & $32,0-37,0$ \\
\hline Fêmea & 7 & $3,0-8,0$ & $31,6-36,9$ \\
\hline \multicolumn{4}{|l|}{ PNMBB } \\
\hline Macho & 2 & $5,0-11,0$ & $32,3-34,5$ \\
\hline \multicolumn{4}{|l|}{ PNMF } \\
\hline Macho & 11 & $2,0-9,0$ & $31,5-36,1$ \\
\hline Fêmea & 1 & 5,0 & 33,9 \\
\hline \multicolumn{4}{|l|}{ PNMCM } \\
\hline Fêmea & 3 & $4,0-6,0$ & $32,0-34,9$ \\
\hline Total & 28 & - & - \\
\hline
\end{tabular}

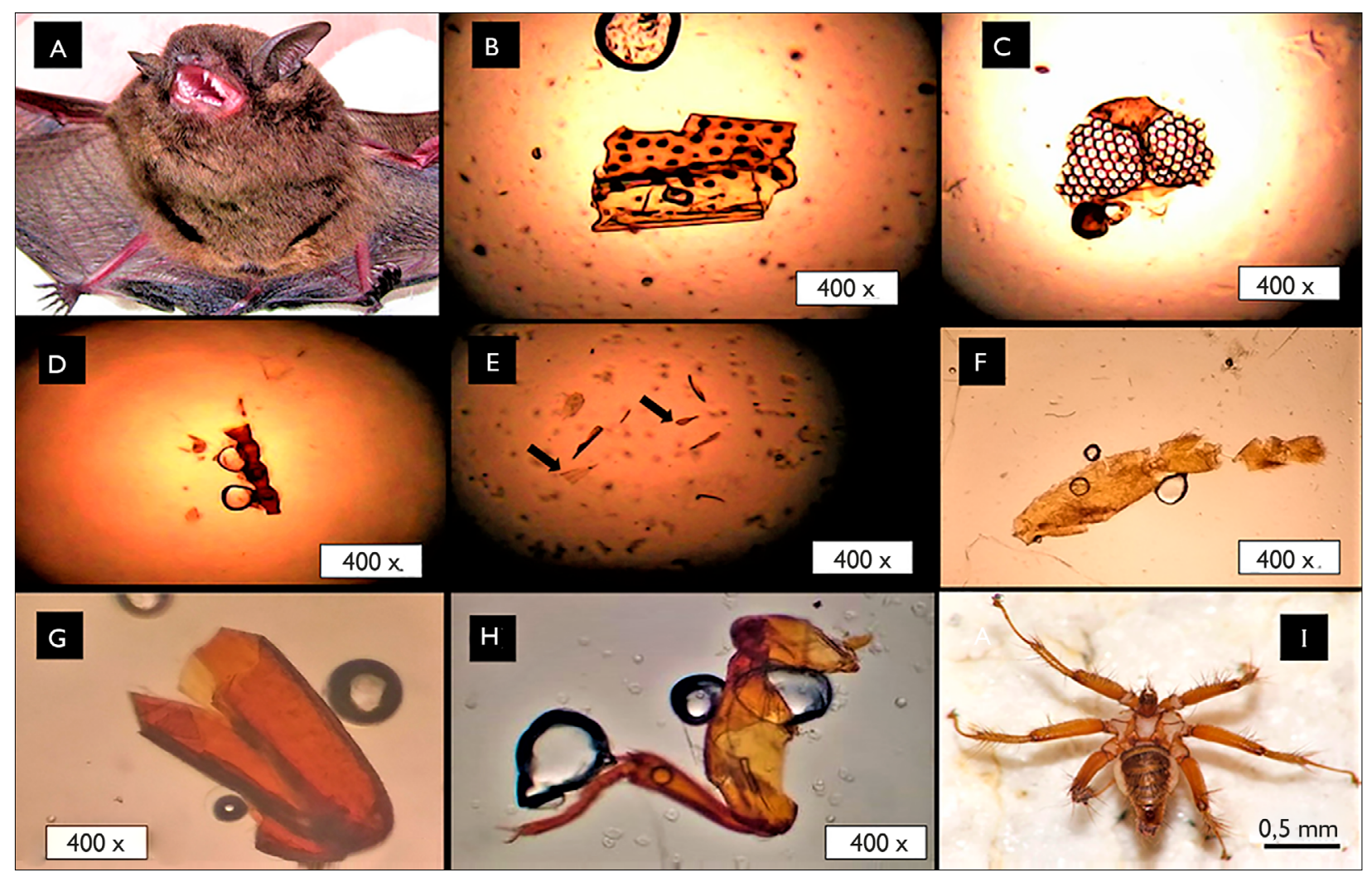

Figura 3. A) indivíduo da espécie Myotis nigricans; B) fragmento do hemiélitro de Hemiptera; C) olho composto (Diptera); D) fragmento de antena (Coleoptera); E) escamas de Lepidoptera; F) fragmento de perna (Diptera) (400x); G) fragmento de perna (Coleoptera); H) perna de Coleoptera; I) Basilia sp. (Diptera: Nycteribiidae). Foto: A: Shirley S. P. Silva (2010); B-H: Raissa S. D. Oliveira (2018); I: Juliana C. Almeida (2010)

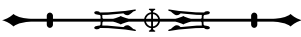




\section{DISCUSSÃO}

\section{DIETA E HISTÓRIA NATURAL}

Os dados biométricos de $M$. nigricans apresentados neste estudo estão de acordo com a literatura, excetuando um macho capturado no PEPB e uma fêmea capturada no PNMCM, que apresentaram peso maior. A partir das medidas de antebraço obtidas nos exemplares coletados, não é possível observar variação nas medidas entre machos e fêmeas que caracterize dimorfismo sexual. No que diz respeito à dieta, a presença de fragmentos de Diptera, Coleoptera, Hemiptera e Lepidoptera nas amostras fecais analisadas corroboram o hábito alimentar insetívoro para a espécie (e.g., Wilson \& LaVal, 1974; LaVal \& Fitch, 1977; Findley, 1993; Aguiar \& Antonini, 2008), embora os dados não sejam suficientes para se propor uma preferência por determinados grupos de insetos. A dieta apresentada permite demonstrar que essa espécie desempenha um serviço ecossistêmico fundamental no controle destas populações de invertebrados (ver Kunz et al., 2011). O estudo de Novaes et al. (2015) sobre M. nigricans na Reserva Ecológica de Guapiaçu (Cachoeiras de Macacu, Rio de Janeiro) registrou indício de frugivoria na dieta de $M$. nigricans, ao relatar a presença de sementes nas fezes dos indivíduos capturados. No presente estudo, não foram observadas sementes ou outras estruturas vegetais nas amostras fecais analisadas.

O baixo número de indivíduos capturados em redes de neblina dificulta o acompanhamento do ciclo reprodutivo das espécies de Myotis nas áreas de estudo. No entanto, os dados indicam que indivíduos de $M$. nigricans apresentam uma variação do padrão reprodutivo ao longo de sua distribuição geográfica, que pode estar relacionada a fatores ambientais como temperatura e disponibilidade de alimento (Racey \& Entwistle, 2000; Rodrigues, 2004). A presença de fêmeas grávidas de $M$. nigricans no mês de outubro confirma o registro obtido por Mottin et al. (2018) no estado de Santa Catarina. Embora nosso estudo contemple 25 anos de amostragens, poucos indivíduos de $M$ nigricans foram capturados, informando que a espécie não é abundante e, talvez, não forme grandes colônias. Portanto, não foi possível determinar o padrão reprodutivo da espécie dentro das áreas inventariadas.

\section{PARASITOLOGIA E CONSERVAÇÃO}

Myotis nigricans é hospedeiro de ácaros, carrapatos, pulgas e moscas (Wilson \& LaVal, 1974). Os espécimes de $M$. nigricans apresentaram em sua pelagem moscas ectoparasitas identificadas como Basilia sp. (Nycteribiidae). Essa associação foi registrada em diversos estudos no Brasil (Graciolli \& Carvalho, 2001; Graciolli \& Aguiar, 2002; Bertola et al., 2005; Graciolli \& Bianconi, 2007; Camilotti et al., 2010), e há pelo menos seis espécies deste gênero infestando $M$. nigricans no país (Graciolli, 2001). Para o estado do Rio de Janeiro, a associação Basilia spp. e Myotis spp. foi registrada por Patrício et al. (2016), ao coletarem exemplares do gênero no município de Seropédica, Rio de Janeiro. No município do Rio de Janeiro, Rio de Janeiro, Almeida et al. (2010) relatam a associação entre Basilia andersoni (Peterson \& Maa, 1970) e M. nigricans, em estudo na Fazenda Marambaia, área localizada a 4,3 km do Parque Estadual da Pedra Branca (PEPB).

Morcegos estão associados a zoonoses causadas por vários tipos de agentes etiológicos, como protozoários, vírus, bactérias e fungos (Bredt et al., 1998; Moratelli \& Calisher, 2015), além de uma grande variedade de endoparasitos, incluindo protozoários, trematódeos, cestódeos e nematódeos (Ubelaker, 1970; Ubelaker et al., 1979; Cuartas-Calle \& Muñoz-Arango, 1999; Santos \& Gibson, 2015). Segundo Coggins (1988), a ocorrência de parasitos pode ser afetada pelo hábito alimentar dos morcegos. A presença de $O$. diminutum (trematódeo digenético) no trato gastrointestinal dos exemplares de M. nigricans estudados pode estar associada à sua dieta insetívora, pois o ciclo da maioria dos trematódeos inclui insetos como hospedeiros intermediários (Ubelaker, 1970; Coggins, 1988; García-Vargas \& Pérez-Ponce De León, 1996). Contudo, não se pode descartar a possibilidade 
desta infestação estar associada à ingestão de água, pois, de acordo com Pérez-Ponce De León (2001), quanto maior o contato do hospedeiro com a água, maior a probabilidade de infecção por estes helmintos. Segundo Ferreira \& Brasil-Sato (1998), esta espécie encontrada em associação com os $M$. nigricans do PEPB é um helminto também encontrado em outros hospedeiros insetívoros, tais como os morcegos da família Molossidae Molossus molossus (Pallas, 1766) e Tadarida brasiliensis (I. Geoffroy, 1824), e os Vespertilionidae Eptesicus fuscus (Beauvois, 1796) e Lasiurus intermedius $\mathrm{H}$. Allen, 1862. O registro de O. diminutum e de um cestódeo no trato intestinal de $M$. nigricans proveniente do PEPB é importante, pois reforça a necessidade de estudos de morcegos urbanos e suas inter-relações com o ambiente.

O levantamento da quiropterofauna de parques urbanos é fator relevante não somente para conhecer a biodiversidade que habita as cidades, mas também para compreender as interações destes animais em ambientes compartilhados com humanos. A partir dos dados apresentados, conclui-se que Myotis nigricans ocupa esse espaço de maneira bem-sucedida nas quatro unidades de conservação: Parque Estadual da Pedra Branca, Parque Natural Municipal da Freguesia, Parque Natural Municipal Chico Mendes e Parque Natural Municipal Bosque da Barra. A despeito dos desafios de viver em ambientes antropizados, não foram encontradas entre os animais capturados evidências de doenças ou prejuízos ao seu desenvolvimento que sejam visíveis e/ou mensuráveis.

\section{AGRADECIMENTOS}

Aos biólogos Alexandre M. S. Carneiro e Joyce S. Damascena, ao médico veterinário Clayton Bernardinelli Gitti e ao Sr. Ricardo P. da Rocha, pelo auxílio nos trabalhos de campo. À Daniela Dias, pela confirmação da identificação dos morcegos, e ao Sérgio Moreira, pela elaboração do mapa. Agradecemos as importantes contribuições de Susi Missel Pacheco e de uma revisora anônima, cujos comentários e sugestões aprimoraram a qualidade do manuscrito.

\section{REFERÊNCIAS}

AGUIAR, L. M. S \& Y. ANTONINI, 2008. Diet of two sympatric insectivores bats (Chiroptera: Vespertilionidae) in the Cerrado of Central Brazil. Revista Brasileira de Zoologia 25(1): 28-31. DOI: http://doi.org/10.1590/S0101-81752008000100005

AGUIRRE, L. F., A. HERREL, R. VAN DAMME \& E. MATTHYSEN, 2003. The implications of food hardness to diet in bats. Functional Ecology 17(2): 201-212. DOI: http://doi.org/10.1046/j.13652435.2003.00721.x

ALMEIDA, J., S. SILVA, N. SERRA-FREIRE \& A. PERACCHI, 2010. Diversidade ectoparasitológica em morcegos na Fazenda Marambaia, Rio de Janeiro, RJ, Brasil. Chiroptera Neotropical 16(Supl.1): 118-121.

BAPTISTA, M. \& C. E. L. ESBÉRARD, 1997. Valores hematológicos de Artibeus sp. e Desmodus rotundus (Mammalia, Chiroptera). Revista Científica Instituto de Pesquisa Gonzaga da Gama Filho 3(2): 11-22.

BERTOLA, P. B., C. C. AIRES, S. E. FAVORITO, G. GRACIOLLI, M. AMAKU \& R. P. ROCHA, 2005. Bat flies (Diptera: Streblidae, Nycteribiidae) parasitic on bats (Mammalia: Chiroptera) at Parque Estadual da Cantareira, São Paulo, Brazil: parasitism rates and hostparasite associations. Memórias do Instituto Oswaldo Cruz 100(1): 25-32. DOI: http://doi.org/10.1590/S0074-02762005000100005

BREDT, A. \&W. UIEDA, 1996. Bats from urban and rural environments of the Distrito Federal, mid-western Brazil. Chiroptera Neotropical 2(2): 54-57.

BREDT, A., F. A. A. ARAUJO, J. CAETANO-JR., M. G. R. RODRIGUES, M. YOSHIKAWA \& M. M. S. SILVA, 1998. Morcegos em áreas urbanas e rurais: manual de manejo e controle. Fundação Nacional de Saúde, Brasília.

CAMILOTTI, V. L., G. GRACIOLLI, M. M. WEBER, J. L. S. ARRUDA \& N. C. CÁCERES, 2010. Bat flies from the deciduous Atlantic Forest in southern Brazil: Host-parasite relationships and parasitism rates. Acta Parasitologica 55(2): 194-200. DOI: https://doi.org/10.2478/ s11686-010-0026-2

CARRERA, M., 1980. Entomologia para você: 5. ed.: 1-185. Nobel, São Paulo.

COGGINS, J. R., 1988. Methods for the ecological study of bat endoparasites. In: T. H. KUNZ (Ed.): Ecological and behavioral methods for the study of bats: 475-489. Smithsonian Institute, Washington.

CUARTAS-CALLE, C. \& J. MUÑOZ-ARANGO, 1999. Nemátodos en la cavidad abdominal y el tracto digestivo de algunos murciélagos Colombianos. Caldasia 21: 10-25. DOI: http://doi.org/10.15446/caldasia

DIAS, D., A. L. PERACCHI \& S. S. P. SILVA, 2002. Quirópteros do Parque Estadual da Pedra Branca, Rio de Janeiro, Brasil (Mammalia, Chiroptera). Revista Brasileira de Zoologia 19(Supl. 2): 113-140. DOI: http://doi.org/10.1590/S0101-81752002000600012

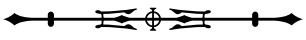


DIAS, D. \& A. L. PERACCHI, 2008. Quirópteros da Reserva Biológica do Tinguá, estado do Rio de Janeiro, sudeste do Brasil (Mammalia: Chiroptera). Revista Brasileira de Zoologia 25(2): 333-369. DOI: http://doi.org/10.1590/S0101-81752008000200023

DIAS, D., S. N. PEREIRA, A. C. S. MAAS, M. A. MARTINS, D. P. BOLZAN \& A. L. PERACCHI, 2010. Quirópteros das regiões Centro-Sul e Médio Paraíba do estado do Rio de Janeiro (Mammalia, Chiroptera). Chiroptera Neotropical 16(1): 579-585.

ESBÉRARD, C. E. L., 2003. Diversidade de morcegos em área de Mata Atlântica regenerada no Sudeste do Brasil. Revista Brasileira de Zoociências 5(2): 189-204.

ESBÉRARD, C. E. L., J. LUZ, L. M. COSTA \& H. G. BERGALLO, 2014. Bats (Mammalia, Chiroptera) of an urban park in the metropolitan area of Rio de Janeiro, southeastern Brazil. Iheringia, Série Zoologia 104(1): 59-69. DOI: http://doi.org/10.1590/16784766201410415969

FERREIRA, V. S. \& M. C. BRASIL-SATO, 1998. Digenéticos (Cercomeria, Lecithodendriidae) de morcegos (Chiroptera) dos municípios de Seropédica e Rio de Janeiro, do estado do Rio de Janeiro, Brasil. Bios 6(6): 83-88.

FINDLEY, J., 1993. Bats: a community perspective. Cambridge University Press (Cambridge Studies in Ecology), Cambridge.

GARCÍA-VARGAS, F. D. \& G. PÉREZ-PONCE DE LEÓN, 1996. Helminth parasites of bats (Mormoopidae and Phyllostomidae) from the Estación de Biología Chamela, Jalisco State, México. Bat Research News 37: 7-8.

GRACIOLLI, G., 2001. Distribuição geográfica e hospedeiros quirópteros (Mammalia, Chiroptera) de moscas nicteribidas americanas (Diptera, Nycteribiidae). Revista Brasileira de Zoologia 18(Supl. 1): 307-322. DOI: http://doi.org/10.1590/S010181752001000500026

GRACIOLLI, G. \& C. J. B. CARVALHO, 2001. Moscas ectoparasitas (Diptera, Hippoboscoidea, Nycteribiidae) de morcegos (Mammalia: Chiroptera) do estado do Paraná, Brasil. II. Streblidae. Chave pictórica para os gêneros e espécies. Revista Brasileira de Zoologia 18(Supl. 1): 907-960. DOI: http://doi.org/10.1590/S010181752001000500002

GRACIOLLI, G. \& L. S. AGUIAR, 2002 Ocorrência de moscas ectoparasitas (Diptera, Streblidae e Nycteribiidae) de morcegos (Mammalia, Chiroptera) no Cerrado de Brasília, Distrito Federal, Brasil. Revista Brasileira de Zoologia 19(Supl. 1): 177-181. DOI: http://doi.org/10.1590/S0101-81752002000500012

GRACIOLLI, G. \& G. V. BIANCONI, 2007. Moscas ectoparasitas (Diptera, Streblidae e Nycteribiidae) em morcegos (Mammalia, Chiroptera) em área de Floresta com Araucária no estado do Paraná, sul do Brasil. Revista Brasileira de Zoologia 24(1): 246-249. DOI: http://doi.org/10.1590/S0101-81752007000100033
GUERRERO, G., 1993. Catalogo de los Streblidae (Diptera: Pupipara) parasitos de murcielagos (Mammalia: Chiroptera) del Nuevo Mundo. I. Clave para los generos y Nycterophilinae. Acta Biologica Venezuelica 14(4): 61-75.

INSTITUTO BRASILEIRO DE GEOGRAFIA E ESTATÍSTICA(IBGE), 2012. Manual técnico da vegetação brasileira: 2. ed. IBGE (Manuais Técnicos em Geociências), Rio de Janeiro.

INSTITUTO ESTADUAL DO AMBIENTE (INEA), 2015. Atlas das Unidades de Conservação do Estado do Rio de Janeiro. Available at: http://www.inea.rj.gov.br/cs/idcplg?IdcService=GET_ FILE\&dID=133385\&dDocName. Accessed on: May 2019.

KUNZ, T. H., E. B. TORREZ, D. BAUER, T. LOBOVA \& T. H. FLEMING, 2011. Ecosystem services provided by bats. Annals of the New York Academy of Sciences 1223: 1-38. DOI: http://doi. org/10.1111/j.1749-6632.2011.06004.x

LAVAL, R. K. \& H. S. FITCH, 1977. Structure, movement, and reproduction in three Costa Rican bat communities. Occasional Papers of the Museum of Natural History 69: 1-28. DOI: http:// doi.org/10.5962/bhl.part.24794

LIMA, I. P., 2008. Espécies de morcegos (Mammalia, Chiroptera) registradas em parques nas áreas urbanas do Brasil e suas implicações no uso deste ambiente. In: N. R. REIS, A. L. PERACCHI \& G. A. S. D. SANTOS (Ed.): Ecologia de morcegos: 71-85. Technical Books Editora, Londrina.

MODESTO, T. C., F. S. PESSÔA, M. C. ENRICI, N. ATTIAS, T. JORDÃO-NOGUEIRA, L. M. COSTA, H. G. ALBUQUERQUE \& H. G. BERGALLO, 2008a. Mamíferos do Parque Estadual do Desengano, Rio de Janeiro, Brasil. Biota Neotropica 8(4): 152-159. DOI: http://doi.org/10.1590/S1676-06032008000400015

MODESTO, T. C., F. S. PÊSSOA, T. JORDÃO-NOGUEIRA, M. C. ENRICI, L. M. COSTA, N. ATTIAS, J. ALMEIDA, D. S. L. RAÍCES, H. G. AlBuQUeRQUe, B. C. PEREIRA, C. E. L. ESBÉRARD \& H. G. BERGALLO, 2008b. Mammals, Serra da Concórdia, state of Rio de Janeiro, Brazil. Check List 4(3): 341-348. DOI: http://doi. org/10.15560/4.3.341

MORATELLI, R. \& C. H. CALISHER, 2015. Morcegos e vírus zoonóticos: podemos associar morcegos com vírus mortais emergentes? Memórias do Instituto Oswaldo Cruz 110(1): 1-22. DOI: http://doi.org/10.1590/0074-02760150048

MOTTIN, V., C. FERNANDO, D. A. S. BÔLLA, J. M. D. MIRANDA, T. S. MACHADO \& F. C. PASSOS, 2018. Atividade reprodutiva e estrutura populacional de Myotis nigricans (Schinz, 1821) (Chiroptera: Vespertilionidae) no sul do Brasil. Mastozoología Neotropical 25(2): 399-406. DOI: http://doi.org/10.31687/saremMN.18.25.2.0.20

NOGUEIRA, M. R., I. P. LIMA, G. S. T. GARBINO, R. MORATELLI, V. C. TAVARES, R. GREGORIN \& A. L. PERACCHI, 2018. Updated checklist of Brazilian bats: versão 2018.1. Comitê da Lista de Morcegos do Brasil-CLMB/Sociedade Brasileira para o Estudo de Quirópteros. Disponível em: https://www.sbeq.net/lista-deespecies. Acesso em: janeiro 2019.

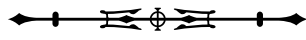


NOVAES, R. L. M., R. F. SOUZA, E. A. RIBEIRO, A. C. SIQUEIRA, A. V. GRECO \& R. MORATELLI, 2015. First evidence of frugivory in Myotis (Chiroptera, Vespertilionidae, Myotinae). Biodiversity Data Journal 3: e6841. DOI: http://doi.org/10.3897/BDJ.3.e6841

PATRÍCIO, P. M. P., E. C. LOURENÇO \& K. M. FAMADAS, 2016. Artrópodes hematófagos de morcegos em refúgios urbanos no estado do Rio de Janeiro. Revista Brasileira de Medicina Veterinária 38(Supl. 3): 157-164.

PERACCHI, A. L. \& M. R. NOGUEIRA, 2010. Métodos de captura de quirópteros em áreas silvestres. In: N. R. REIS, A. L. PERACCHI, B. K. ROSSANEIS \& M. N. FREGONEZI (Ed.): Técnicas de estudos aplicadas aos mamíferos silvestres brasileiros: 1. ed.: 42-58. Technical Books, Rio de Janeiro.

PÉREZ-PONCE DE LEÓN, G., 2001. Diversity of Digeneans (Platyhelminthes: Cercomeria: Trematoda) the in vertebrates in Mexico. Comparative Parasitology 68(1): 1-8.

PINTO, A. C. D. C., 2008. Comunidade de quirópteros (Mammalia, Chiroptera) do Parque Natural Municipal da Prainha, Rio de Janeiro, RJ, Brasil. Masther Thesis - Universidade Federal Rural do Rio de Janeiro, Seropédica.

RACEY, P. A. \& A. C. ENTWISTLE, 2000. Life-history and reproductive strategies of bats. In: E. G. CRICHTON \& F. P. KRUTZSCH (Ed.): Reproductive biology of bats: 363-414. Academic Press, Boston.

REIS, N. R., M. N. FREGONEZI, A. L. PERACCHI \& O. K. SHIBATTA, 2013. Morcegos do Brasil: guia de campo. Technical Books, Rio de Janeiro.

RIZZINI, C. T., 1979. Tratado de fitogeografia do Brasil: aspectos sociológicos e florísticos: vol. 2: 1-374. Editora Hucitec, São Paulo.

ROCHA, C. F. D., H. G. BERGALLO, M. A. S ALVES \& M. VAN SLUYS, 2003. A biodiversidade nos grandes remanescentes florestais no estado do Rio de Janeiro e nas restingas da Mata Atlântica. Rima Editora, São Carlos.

RODRIGUES, W. C., 2004. Fatores que influenciam no desenvolvimento dos insetos. Entomologistas do Brasil 1(4): 1-4.
RYDELL, J., 1992. Occurrence of bats in northernmost Sweden $\left(65^{\circ} \mathrm{N}\right)$ and their feeding ecology. Journal of Zoology 227(3): 517-529. DOI: http://doi.org/10.1111/j.1469-7998.1992.tb04412.x

SANTOS, C. P. \& D. L. GIBSON, 2015. Checklist of the helminth parasites of South American bats. Zootaxa 3937(3): 471-499. DOI: http://doi.org/10.11646/zootaxa.3937.3.3

SHIEL, C., C. MCANEY, C. SULLIVAN \& J. FAIRLEY, 1997. Identification of Arthropod Fragments in Bat Droppings. Mammal Society Occasional Publications (17): 1-56.

SILVA, S. S. P., A. P. CRUZ, J. C. ALMEIDA \& A. L. PERACCHI, 2010. Bionomia de morcegos em áreas urbanas: Parque Natural Municipal da Freguesia e Fazenda Marambaia no município do Rio de Janeiro, RJ. Chiroptera Neotropical 16(Supl.): 6-11.

STRAUBE, F. C. \& G. V. BIANCONI, 2002. Sobre a grandeza e a unidade utilizada para estimar esforço de captura com utilização de redes-de-neblina. Chiroptera Neotropical 8: 150-152.

UBELAKER, J. E., 1970. Some observations on ecto and endoparasites of Chiroptera. In: B. H. SLAUGHTER \& D. W. WALTON (Ed.): About bats: 247-261. Southern Methodist University, Dallas, Texas.

UBELAKER, J. E., R. D. SPECIAN \& D. W. DUSZYNSKI, 1979. Endoparasites. In: R. J. BAKER, J. K. JONES JR. \& D. C. CARTER (Ed.): Biology of bats of the new world family Phyllostomatidae: Part III: 7-56. Museum of Texas Tech University (Special Publication 16), Texas.

VIZOTTO, L. D. \& V. A. TADDEI, 1973. Chave para a determinação de quirópteros brasileiros. Revista da Faculdade de Filosofia, Ciências e Letras de São José do Rio Preto 1: 1-72.

WHITAKER, J. O., 1988. Food habits analysis of insectivorous bats. In: T. H. KUNZ (Ed.): Ecological and behavioral methods for the study of bats: 1. ed.: 171-189. Smithsonian Institution Press, Washington.

WILSON, D. E. \& R. K. LAVAL, 1974. Myotis nigricans. Mammalian Species 39: 1-3. DOI: http://doi.org/10.2307/3503847

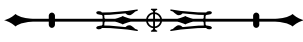

